\title{
A compact temperature insensitive filter using splitter polarization-rotating section
}

\author{
Sarvagya Dwivedi, Herbert D'heer and Wim Bogaerts, Member IEEE \\ Photonics Research Group, Department of Information Technology Ghent University—imec, \\ Center for Nano- and Biophotonics, Sint-Pietersnieuwstraat 41, B-9000 Ghent, Belgium \\ Email: sarvagya.dwivedi@intec.ugent.be
}

\begin{abstract}
A compact all-silicon Mach-Zehnder interferometer (MZI) filter with temperature sensitivity less than $5 \mathrm{pm} / \mathrm{K}$ is demonstrated. The device achieves a reduced footprint by making use of different polarizations.
\end{abstract}

Index Terms - Silicon photonics, wavelength filtering devices, athermal, polarization selective devices

\section{INTRODUCTION}

Silicon has a very high refractive index, enabling optical waveguides with a very high refractive index contrast which opens the possibility of micron size devices in silicon photonics. An important limitation for wide adoption of silicon photonic devices is the high thermo-optic (TO) coefficient of silicon $\left(1.86 \times 10^{-4} \mathrm{~K}^{-1}\right)$. This means that the refractive index changes with temperature and hence needs a tuning to achieve a fixed wavelength operation. Several methods have been proposed to compensate this effect, one of them being the introduction of materials with negative thermo-optic coefficients as cladding, such as polymers [1]. However, this introduces additional process complexity and it is not always desirable to incorporate such materials in a CMOS-like process flow. Another approach is to use local heaters to dynamically stabilize the device but this approach consumes power [2].

For an interferometric device such as a Mach-Zehnder Interferometer (MZI) it is also possible to obtain temperature insensitivity without such measures by combining wide and narrow waveguides in different arms [3, 4]. The two arms of the MZI have different thermo-optic coefficients which directly depend upon the confinement in the silicon core. By choosing appropriate arm lengths the temperature sensitivity of both the arms can be made such to cancel each other. However this technique tends to increase the device footprint considerably. In [4] an alternative approached is proposed to realize temperature insensitivity while keeping a small device footprint. This requires a large difference in the TO coefficient between both arms hence very narrow and wide waveguides but the performance of such a device is then limited by the high propagation loss of narrow waveguides. In this paper we propose to use the TM polarization in a broader waveguide since the TM mode is less confined in the silicon core than the TE mode, and this result in lower TO coefficient. The loss is also lower since the TM mode does not have high field intensities on the etched sidewalls. By this we can make a compact temperature insensitive MZI filter by exploiting both polarizations supported by the on-chip waveguides. The measured device is one-third in footprint with respect to [3].

\section{DESIGN AND FABRICATION}

The device includes a splitter-polarization rotator (SPR) which splits the light into two parts and rotates the polarization in one arm. It's an asymmetrical directional coupler conditioned for phase matching between TE and TM. The compactness of the athermal filter depends upon the difference in TO coefficients of the arms. Figure 1 shows the TO coefficient of TE and TM silicon on insulator (SOI) waveguides as a function of waveguide width.

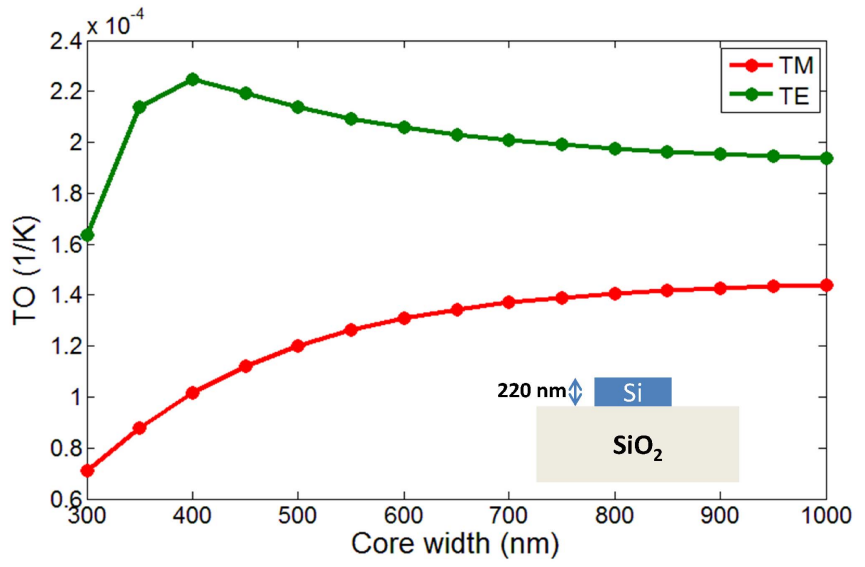

Fig. 1. Thermo-optic coefficients of TE and TM modes of SOI waveguide (inset) with increasing core width

In a standard MZI, the Free Spectral Range (FSR) is defined as

$$
F S R=\frac{\lambda^{2}}{n_{g} \Delta L}
$$

where $\lambda$ is center wavelength, $\mathrm{n}_{\mathrm{g}}$ is the group index and $\Delta \mathrm{L}$ is the path length difference between both arms. For the proposed structure the modified expression is

$$
F S R=\frac{\lambda^{2}}{n_{g p} L_{p}-n_{g s} L_{S}}
$$

where $n_{g p} L_{p}$ and $n_{g s} L_{s}$ correspond to group index and lengths for TM and TE arms respectively. To make the device athermal the wavelength shift with respect to temperature should be brought to zero i.e.

$$
\frac{d \lambda}{d T}=\frac{\lambda\left(\frac{d n_{e f f_{T M}}}{d T} \cdot L_{p}-\frac{d n_{e f f_{T E}}}{d T} \cdot L_{S}\right)}{n_{g p} L_{p}-n_{g s} L_{S}} \rightarrow 0
$$


Here, $T$ is the temperature and $\frac{d n_{\text {eff }}}{d T}$ is the thermo optic coefficient of the corresponding arms. The fabricated device is shown in figure 2. The input transverse electric (TE) polarized light passes through the SPR that splits and converts half of the power in TM polarization going to the upper arm, while the remaining half of the input power remains in the lower arm as TE polarized light. Finally, both polarizations combine at the MZI output using a similar SPR as a combiner.

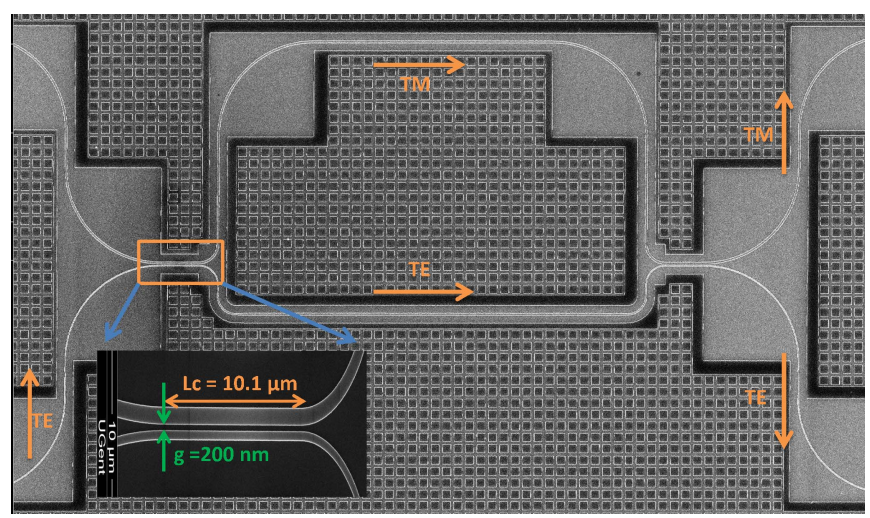

Fig. 2. SEM image of fabricated athermal MZI filter with zoomed in SPR (inset) of coupling length $\mathrm{Lc}$ of $10.1 \mu \mathrm{m}$ and gap of $200 \mathrm{~nm}$.

The device was fabricated in the imec $200 \mathrm{~mm}$ CMOS pilot line, using silicon-on-insulator wafers with $220 \mathrm{~nm}$ of silicon and $2 \mu \mathrm{m}$ of buried oxide.

\section{MEASUREMENTS}

The measured length of the SPR is $10.1 \mu \mathrm{m}$ and the gap is 200 $\mathrm{nm}$. The width of the SPR waveguides is $560 \mathrm{~nm}$ and $310 \mathrm{~nm}$ respectively. The measured width of upper TM arm and lower $\mathrm{TE}$ arm is $560 \mathrm{~nm}$. The reason of making a wider section is to have low loss in the device. Since the upper arm contains TM mode, larger bends with a radius of $25 \mu \mathrm{m}$ are used in the design for better mode confinement and low loss. The simulation of the device is done with in-house circuit simulator Caphe [5]. For measurement we placed the fabricated chip on a thermally controlled chuck with a resistive heater and temperature sensor. The measured and simulated results of device transmission and thermal drift with varying wavelength are shown in figure 3 . The measured thermal sensitivity of the device is less than $8 \mathrm{pm} / \mathrm{K}$ over $28 \mathrm{~nm}$ of wavelength range and compared with simulations its bit narrower due to fabrication variations and waveguide tapering in the fabricated device. The insertion loss at the lower part of wavelength in device is due to the asymmetrical directional couplers. The FSR of the device is changing from $9.5 \mathrm{~nm}$ to $13.5 \mathrm{~nm}$ due to group index variation of the TM waveguide. Because the TO coefficients are a function of wavelength, the device spectrum is red shifted for increasing temperatures for wavelengths lower than the athermal point, while at higher wavelengths the spectrum is blue shifted.

\section{CONCLUSION}

We demonstrated a compact temperature insensitive MZI filter using a splitter with polarization- rotating section.

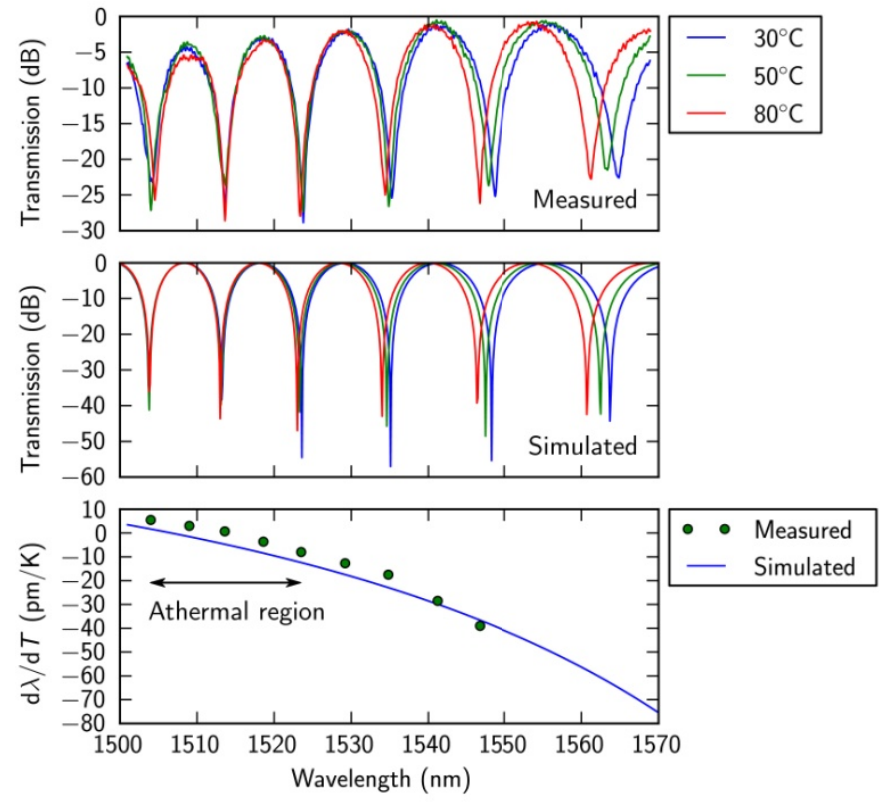

Fig. 3(a) Measured and simulated spectra for 30, 50 and 80 deg. $C$ and in (b) $\mathrm{d} \lambda /$ dT variation with wavelength.

The measured result shows that the temperature insensitivity is less than $5 \mathrm{pm} / \mathrm{K}$ over a wavelength range of $20 \mathrm{~nm}$. The foot-print of the device is one-third of previously reported athermal MZI filter. This device opens a new class of low loss and compact temperature insensitive silicon photonic devices for wavelength division multiplexing applications.

\section{ACKNOWLEDGMENT}

The authors acknowledge the efforts of imec's 200-mm pline for their contributions to the device fabrication, Martin Fiers for help in circuit simulation software Caphe and Michael Vanslembrouck for measurements support. This work was supported by imec's Optical I/O Core Program.

\section{REFERENCES}

[1] J. Teng, P. Dumon, W. Bogaerts, H. Zhang, X. Jian, X. Han, M. Zhao, G. Morthier, and R. Baets, "Athermal Silicon-on-insulator ring resonators by overlaying a polymer cladding on narrowed waveguides," Opt. Exp., vol. 17, pp. 14627-14633 (2009)

[2] H. Yu, M. Pantouvaki, S. Dwivedi, P. Verheyen, G. Lepage, R. Baets, W. Bogaerts, P. Absil, J. Van Campenhout, "Compact, Thermally Tunable Silicon Racetrack Modulators Based on an Asymmetric Waveguide," IEEE photonics technology letters, (2013)

[3] M. Uenuma, and T. Moooka, "Temperature-independent silicon waveguide optical filter," Opt. Lett., vol. 34, pp. 599-601 (2009).

[4] B. Guha, A. Gondarenko, and M. Lipson, "Minimizing temperature sensitivity of silicon Mach-Zehnder interferometers," Opt. Exp., vol. 18, pp. 1879-1887 (2010).

[5] M. Fiers, T. Van Vaerenbergh, K. Caluwaerts, D. Vande Ginste, B. Schrauwen, J. Dambre, P. Bienstman, "Time-domain and frequency-domain modeling of nonlinear optical components on circuit-level using a node-based approach," Journal of the Optical Society of America B, 29(5), p.896-900 (2012) 